\title{
Análisis de rentabilidad en la etapa de diseño entre un modelo bidimensional CAD y un modelo BIM para el proyecto de Intercambio Vial, Km 25+115.85 de la Autopista Juliaca - Puno
}

Profitability analysis in the design stage between a two-dimensional CAD model and a BIM model for the Road Interchange project, $\mathrm{Km} 25+115.85$ of the Juliaca - Puno Highway

Análise de rentabilidade em fase de projeto entre um modelo CAD bidimensional e um modelo BIM para o projeto de Trevo Rodoviário, Km 25 + 115,85 da Rodovia Juliaca - Puno

\section{ARTÍCULO GENERAL}

sergiollanos@,outlook.com.pe https://orcid.org/0000-0002-2252-4708

Universidad Peruana Unión Lima, Perú
Sujei Ñaupa Mamani

sujei.mamani@gmail.com https://orcid.org/0000-0002-5304-4782

Universidad Peruana Unión Lima, Perú
Samuel Marca Arocutipa

samuelmarcaarocutipa@gmail.com https://orcid.org/0000-0002-0423-853X

Universidad Peruana Unión Lima, Perú

Correspondencia: Rubén Fitzgerald Sosa Aquise

https://orcid.org/0000-0003-1058-8285; ruben.sosa@upeu.edu.pe

Recibido 07 de Abril 2021 | Arbitrado y aceptado 19 de Junio 2021 | Publicado en 03 Setiembre 2021

\section{RESUMEN}

El presente artículo tiene como objetivo el estudio de la rentabilidad que produce la aplicación de la tecnología Buildig Information Modeling (BIM), en la etapa de diseño del expediente, frente al método tradicional de cálculo que se fundamenta en dibujos en dos dimensiones (2D). El caso de estudio es la estructura formada por planchas de acero corrugado galvanizado (Multiplate MP152), cimentación y muros de concreto armado, relleno estructural y pavimento flexible, del proyecto de Intercambio Vial,

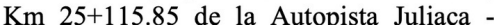
Puno. Se presenta la metodología para el desarrollo del modelo BIM teniendo en cuenta cinco variables (dimensión en el eje $\mathrm{x}$, dimensión en el eje $\mathrm{y}$, dimensión en el eje $\mathrm{z}$, tiempo y costo), con la integración de información utilizando herramientas como software de Autodesk: Recap, Civil 3D, Revit, Infrawork y Navisworks. Se efectúa una comparación del Diseño de las partidas más importantes, obtenido con el método tradicional y con la utilización de BIM, a partir de la cual es posible verificar el aporte y/o rentabilidad que produce la implementación de BIM en el diseño de estas partidas. Como resultado de la modelación en BIM, se valora un ahorro de $24 \%$ en cuanto al tiempo de ejecución y en función de la rentabilidad se estima que dicho ahorro con BIM es de $43.81 \%$ con relación al $\mathrm{CAD}$ concluyendo que la tecnología BIM es más sencilla y eficaz de gestionar, agilizando todos los procesos de diseño.

Palabras claves: BIM, CAD, rentabilidad, intercambio vial, planchas de acero corrugado galvanizado Multiplate MP152, herramientas de software.

\section{ABSTRACT}

The objective of this article is to study the profitability produced by the application of Buildig Information Modeling (BIM) technology, in the file design stage, compared to the traditional calculation method that is based on two-dimensional drawings (2D). The case study is the structure formed by galvanized corrugated steel plates (Multiplate MP152), reinforced concrete foundations and walls, structural fill and flexible pavement, of the Road Interchange project, $\mathrm{Km} 25+115.85$ of the Juliaca - Puno Highway. The methodology for the development of the BIM model is presented taking into account five variables (dimension on the $\mathrm{x}$ axis, dimension on the $\mathrm{y}$ axis, dimension on the $\mathrm{z}$ axis, time and cost), with the integration of information using tools such as Autodesk software : Recap, Civil 3D, Revit, Infrawork and Navisworks. A comparison is made of the Design of the most important items, obtained with the traditional method and with the use of BIM from which it is possible to verify the contribution and / or profitability produced by the implementation of BIM in the design of these items. As a result of the modeling in BIM, a saving of $24 \%$ in terms of execution time is valued and based on profitability it is estimated that said saving with $\mathrm{BIM}$ is $43.81 \%$ in relation to $\mathrm{CAD}$, concluding that BIM technology is simpler and efficient to manage, streamlining all design processes.

Keywords: BIM, CAD, profitability, road interchange, Multiplate MP152 galvanized corrugated steel sheets, software tools.

\section{RESUMO}

O objetivo deste artigo é estudar a rentabilidade produzida pela aplicação da tecnologia Buildig Information Modeling (BIM), na etapa de projeto de arquivos, em comparação ao método tradicional de cálculo baseado em desenhos bidimensionais (2D). O estudo de caso é a estrutura formada por chapas de aço galvanizado ondulado (Multiplate MP152), fundações e paredes de concreto armado, preenchimento estrutural e pavimento flexível, do projeto Trevo Rodoviário, Km $25+115,85$ da Rodovia Juliaca - Puno. A metodologia de desenvolvimento do modelo BIM é apresentada levando em consideração cinco variáveis (dimensão no eixo $\mathrm{x}$, dimensão no eixo $\mathrm{y}$, dimensão no eixo $\mathrm{z}$, tempo e custo), com integração de informações por meio de ferramentas como o Autodesk softwares: Recap, Civil 3D, Revit, Infrawork e Navisworks. É feita uma comparação do Desenho dos itens mais importantes, obtidos com o método tradicional e com o uso do BIM, a partir do qual é possível verificar a contribuição e / ou rentabilidade produzida pela implementação do BIM no desenho desses itens. . Como resultado da modelagem em BIM, avalia-se uma economia de $24 \%$ em termos de tempo de execução e com base na rentabilidade estima-se que essa economia com BIM seja de $43,81 \%$ em relação ao $\mathrm{CAD}$, concluindo que a tecnologia BIM é mais simples e eficiente para gerenciar, agilizando todos os processos de design.

Palavras-chave: BIM, CAD, lucratividade, intercâmbio rodoviário, chapas de aço galvanizado galvanizado Multiplate MP152, ferramentas de software. 


\section{Introduccion}

BIM es una metodología colaborativa para la gestión de proyectos en obras civiles que aporta a los proyectos gran rentabilidad, eficiencia y sostenibilidad ya que genera un ahorro económico que ejercerá una mejor colaboración entre las diferentes disciplinas del diseño y ejecución de una obra de carretera. BIM, afirma Borja (2015), centraliza toda la información de un proyecto (3D o geométrica, 4D o tiempos, 5D o costes, 6D o ambiental, y 7D o mantenimiento) en un modelo digital desarrollado por todos sus agentes, permitiendo ahorros de tiempo y costos en un proyecto constructivo.

La situación según ASIDEK (2016) del BIM en el mundo ha ido evolucionando exponencialmente, con un crecimiento de la participación económica de países como EUA, Canadá, Reino Unido, Alemania o Francia, que ya apuestan por integrar BIM en su estrategia dentro del sector de arquitectura, ingeniería, construcción y operación (AECO). Se estima que para 2020, el mercado BIM crecerá hasta un $12 \%$ en Norte América, 13\% en Europa y Asia, y 11\% en el resto del mundo. Inicialmente, BIM fue diseñado para ser aplicado en el sector de la construcción, pero se está expandiendo a otras áreas de la construcción para las que no fue designado originalmente, como la infraestructura civil. Se cree que el uso de BIM en infraestructura está atrasado casi tres años con respecto a su uso para edificios, pero la evidencia muestra que recientemente el uso de BIM en infraestructura está aumentando

Fernando (2017), menciona que una de las ventajas que trae la utilización de este software es que se puede generar topografía desde las imágenes satelitales disponibles en sus servidores, esto permite tener una idea general de la superficie en donde se desarrollará el proyecto. La elaboración del diseño se muestra en forma automatizada, la visualización del proyecto se muestra en un plano 3D y que además contiene la información de cada uno de los componentes por los que están constituidos el proyecto. El software tiene la capacidad de extraer del modelo de manera automática la medición de todos ellos (Chavarria, 2018). 
Tabla 1. Diferencia BIM y CAD

\begin{tabular}{|c|c|c|}
\hline CONCEPTO & CAD & BIM \\
\hline Dibujo & $\begin{array}{l}\text { Se realiza por entidades } \\
\text { geométricas como líneas, } \\
\text { círculos, polígonos, sólidos y } \\
\text { superficies }\end{array}$ & $\begin{array}{l}\text { Se realiza con elementos } \\
\text { constructivos con propiedades } \\
\text { como muros, pilares, ventanas, } \\
\text { cubiertas, terrenos. }\end{array}$ \\
\hline $\begin{array}{l}\text { Relación } \\
\text { plantas,modelos, } \\
\text { alzados. }\end{array}$ & $\begin{array}{l}\text { Son entidades independientes y } \\
\text { hay que aplicar cambios por } \\
\text { separado, dado que los archivos } \\
\text { son independientes entre sí. }\end{array}$ & $\begin{array}{l}\text { Existe un único modelo del que } \\
\text { se extraen representaciones, } \\
\text { cualquier cambio en el modelo } \\
\text { cambia las representaciones }\end{array}$ \\
\hline Datos asociados & $\begin{array}{l}\text { Bloques con atributos( Poco } \\
\text { utilizados, tiene limitaciones) }\end{array}$ & $\begin{array}{l}\text { Los elementos cuentan con } \\
\text { propiedades. }\end{array}$ \\
\hline Informes & $\begin{array}{l}\text { Calcular datos y exportarlos a } \\
\text { otros softwares ( Excel) }\end{array}$ & $\begin{array}{l}\text { Son generados } \\
\text { automáticamente y tienen } \\
\text { conexión directa entre ellos }\end{array}$ \\
\hline $\begin{array}{l}\text { Trabajo en } \\
\text { grupo }\end{array}$ & $\begin{array}{l}\text { No hay, soluciones } \\
\text { improvisadas, un archivo una } \\
\text { persona. }\end{array}$ & $\begin{array}{l}\text { Posibilidad de trabajo e grupo } \\
\text { sobre un mismo modelo, existe } \\
\text { coherencia en el diseño final. }\end{array}$ \\
\hline
\end{tabular}

Nota. Tomado de Monfort (2015).

BIM se aplica principalmente en la generación y gestión de datos de edificios.

Utiliza el concepto orientado a objetos para aumentar la eficiencia de la gestión de la información en el ciclo de vida del edificio" (Noor \& Yi, 2017). Huang (como se cita en Noor \& Yi, 2017) demostró que BIM puede simplificar el diseño y la construcción de la alineación de vías, aumentando las capacidades del diseño asistido por computadora y la automatización, lo que acorta en gran medida el período de diseño y aumenta la eficiencia de la construcción. El BIM permite una inimaginable gama de actividades colaborativas, revisión interdisciplinaria e integrada del diseño, coordinación multimodelos y detección de interferencias, integración en tiempo real con otros especialistas para la estimación de costos, gestión de la construcción, etc" (Enshassi \& AbuHamra, 2016; Karlshøj, 2012).

Entre las herramientas que permiten desarrollar este modelo digital, se destaca AutoCAD Civil 3D Software de diseño lineal de proyectos de infraestructura (EquipoPVP, 2018). En AutoCAD Civil 3D se puede establecer flujos de trabajo, se pueden desarrollar proyectos civiles, infraestructura, como distribución lotes, trazo de redes de abastecimiento de agua potable, alcantarillado, drenaje, vías, canales, además se puede procesar estudios topográficos (Huancas y Torres, 2020). Actualmente se puede utilizar con uno de los softwares más potentes que la empresa de Autodesk, 
InfraWorks, este software puede ser utilizada en las fases como planeación y poder lograr la visualización del proyecto en la etapa final. este software nos permite elaborar modelos exactos en comparación a nuestra realidad y nos posibilita trabajar sobre los mismos de una manera simple y práctica, debido a que cuenta con herramientas muy útiles (Fernando, 2017).

Es importante tener en cuenta que "la industria de la construcción tiene un impacto directo sobre el crecimiento económico de un país. El sector de la construcción ha implementado diferentes metodologías para la gestión de proyectos a lo largo de la historia, pero las diferencias entre los tiempos y los costos se presentan constantemente a nivel mundial. Por otro lado, los proyectos de construcción cada vez son más complejos, lo que aumenta los riesgos y exige la utilización e implementación de nuevas técnicas y herramientas. La metodología BIM (Building Information Modeling por sus siglas en inglés) ha tomado fuerza en los últimos años, demostrando ser eficiente para una adecuada gestión de proyectos.” (Contreras et al., 2018)

La investigación de esta problemática se realizó por el interés de conocer "si el proyecto que implementa BIM, reduce el tiempo de trabajo para estimar los metrados para costos, porque reduce los días de trabajo al realizar el modelado, cuantificar el metrado y realizar la revisión respectiva para cada proyecto" (Medina y Salomón, 2019) y en el ámbito profesional, como ingenieros, el interés versó en conocer el contexto de la metodología BIM y su aplicación en obras de infraestructura vial, conocer las ventajas del modelamiento digital, así como programar, organizar actividades y facilitar la coordinación activa de todos los involucrados en el proyecto, con un intercambio de información y mediante un entorno común de datos.

Por último, el presente artículo tuvo como objetivo principal realizar un análisis de rentabilidad entre dos alternativas para la elaboración del proyecto en la etapa de diseño usando Building Information Modeling (BIM) y usando la metodología del diseño asistido por computadora (CAD). Además, fue necesario realizar lo siguiente: a) Analizar e interpretar los estudios con la metodología convencional CAD, b) Desarrollar el modelo BIM del intercambio vial 25+115.85 - LOD 400, c) Evaluar el rango de variabilidad del índice de rentabilidad entre el modelo convencional CAD y metodología BIM en las dimensiones de tiempos y costos del proyecto de intercambio vial $25+115.85$, d) Analizar las ventajas y desventajas del uso de herramientas de 
gestión de proyectos BIM y el método convencional post implementación en la etapa de expediente técnico.

\section{Materiales y Métodos}

\section{Materiales}

La metodología utilizada para la presente investigación hace referencia al método científico experimental. El desarrollo de la metodología se enfoca en el estudio del impacto de BIM en el cálculo de presupuestos de construcción de estructuras en concreto reforzado, frente al método tradicional de cálculo. Elaborar un presupuesto de obra resulta una labor compleja dada, la magnitud de los proyectos de construcción, las múltiples tareas de obra, la incertidumbre en obra, los factores climáticos, la variedad de los materiales empleados en el proyecto, los variables precios de materia

\subsection{Métodos}

\subsubsection{Analizar e interpretar los estudios con la metodología convencional CAD}

Las empresas constructoras, normalmente desarrollan proyectos que involucran concreto, ya sea desde un simple cimiento hasta muros reforzados y una variedad de tipos de estructuras que tienen inmerso principalmente dicho material pertenecientes a proyectos de diversa envergadura, sin embargo, hasta la actualidad todas estas estructuras siempre han sido plasmadas desde la fase de su idea inicial como proyecto hasta el momento de iniciar su construcción para reconocer cada elemento del proyecto visualmente siempre en dos dimensiones.

En CAD, la cooperación entre los distintos agentes implicados en el proyecto (ingenieros, empresas encargadas de la construcción, proveedores, etc.) no es posible debido a las limitaciones del software. En lo referente a la gestión de la información, en el caso de CAD el peso principal de los archivos creados son planos independientes unos de otros.

El CAD tiene menos precisión en cuanto al cálculo del metrado lo cual tiene un impacto directo en los costos de las obras en comparación a otras metodologías.

Se analizó los estudios básicos y planos de diseño del expediente técnico: Informe de Modificación de Ingeniería de la Variante de Paucarcolla correspondiente a la 
Construcción de la Autopista Puno - Juliaca del Ministerio de Transportes y

Comunicaciones (2016), con ellos se logró establecer cuáles serían las partidas de diseño más importantes.

Tales como la ubicación:
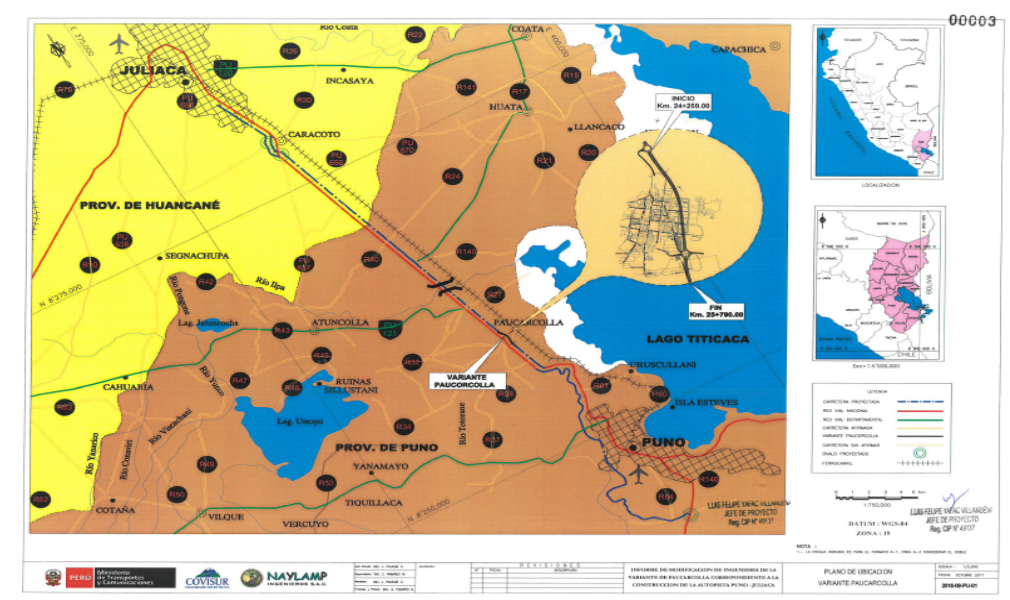

Figura 1. Plano Ubicación Intercambio Vial. Fuente, Expediente Técnico: Informe de Modificación de Ingeniería de la Variante de Paucarcolla correspondiente a la Construcción de la Autopista Puno - Juliaca

Asimismo, se muestra los planos más resaltantes del intercambio vial.
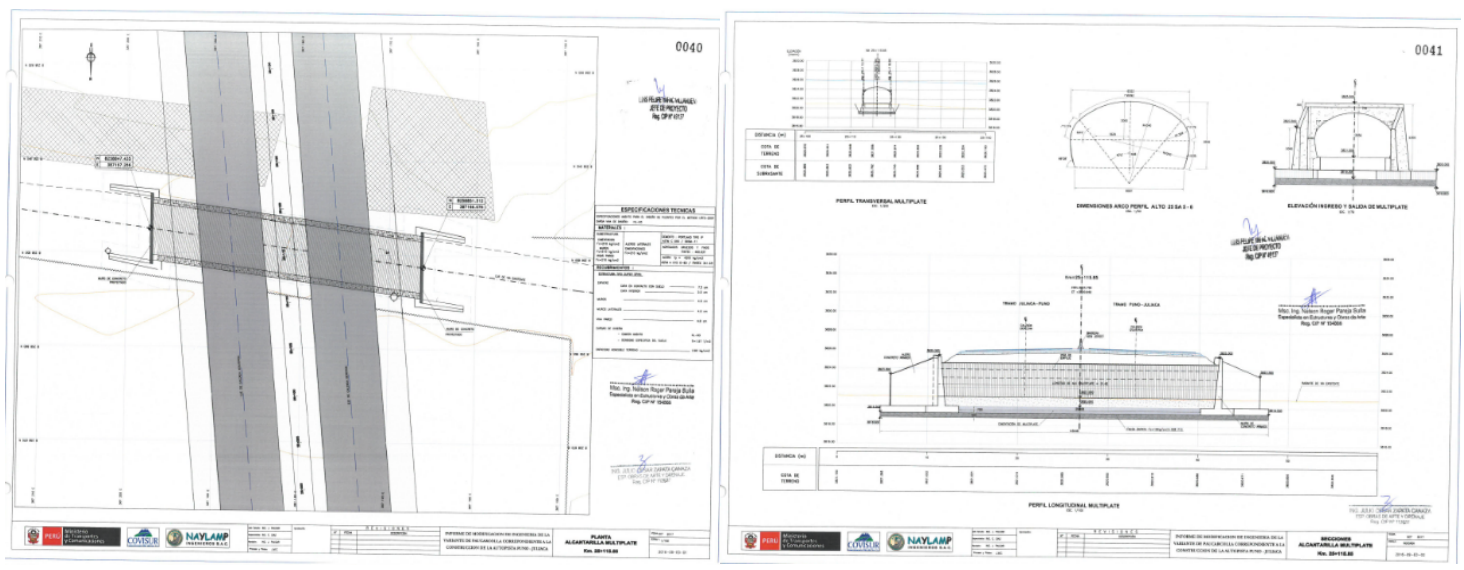

Figura 2. Plano Planta y Sección Intercambio Vial. Fuente, Expediente Técnico:

Informe de Modificación de Ingeniería de la Variante de Paucarcolla correspondiente a la Construcción de la Autopista Puno - Juliaca 


\subsubsection{Desarrollar el modelo BIM del intercambio vial 25+115.85 - LOD 400.}

Teniendo la información contractual de las diferentes especialidades analizadas del expediente técnico y tomando en cuenta los niveles proyectados se realizará el modelamiento BIM utilizando variedad de herramientas disponibles las cuales sirven como apoyo para la realización del proyecto.

\section{Programas informáticos}

En el año 2020 se realizaron diferentes webinar impartidos con el representante Victoria Ureña, en la cual se explicó las herramientas digitales para el desarrollo de proyectos de infraestructuras civiles indicando que existen tres programas clave que ofrecen soluciones complementarias, integradas y en constante evolución para la conceptualización, el diseño detallado y la documentación del proyecto: Autodesk InfraWorks, AutoCAD Civil 3D, Revit Estructural. Estos programas permiten trabajar con metodología BIM y flujos de trabajo conectados. Además, con Dynamo sobre Revit y Civil 3D podemos automatizar tareas repetitivas y reducir los tiempos de diseño.

\section{Niveles de detalle}

Tal como lo plantea el NBS del Reino Unido, el término Level of Definition (nivel de definición) se refiere al nivel de detalle (descripción gráfica de los modelos en cada etapa) y al nivel de información (LOI, que describe el contenido no gráfico del modelo en cada etapa). A continuación, se indican los siguientes niveles de detalle e información, acorde a lo señalado por el BIM Forum Chile (2017).

Tabla 2. Niveles de detalle

\begin{tabular}{ll}
\hline Nivel de detalle & caracteristicas \\
\hline LOD 1 & Conceptualizacion y casi nula geometría \\
LOD 2 & el elemento de construcción modelado proporciona una \\
& indicación visual del elemento en la etapa conceptual, \\
& identificando requerimientos claves como el acceso o zonas \\
& libres para el posterior mantenimiento. Esta información es \\
& adecuada para la coordinación espacial inicial de los elementos \\
& o sistema. \\
el elemento de construcción modelado proporciona una \\
representación visual del elemento en la etapa de definiciones \\
técnicas para su coordinación espacial completa
\end{tabular}


LOD 4

el elemento de construcción modelado proporciona una representación visual del elemento para una etapa de diseño. Con su coordinación espacial completa.

Nota. Tomado de BIM Forum Chile (2017)

\section{Niveles de desarrollo}

Ahora bien, el AIA (American Institute of Architects) de Estados Unidos define al nivel de desarrollo como la forma de identificar requisitos mínimos y usos específicos a cada elemento del modelo, en un respectivo nivel. Los siguientes, son los niveles de desarrollo, en base a lo expuesto por el BIM Forum Chile y desde la definición del AIA (2017):

Tabla 3. Niveles de desarrollo

\begin{tabular}{ll}
\hline Nivel de desarrollo & Características \\
\hline LOD 100 & El elemento puede ser representado gráficamente en el modelo \\
& con un símbolo o representación genérica. Estas \\
& representaciones muestran la existencia de un componente, \\
& pero no su forma, tamaño o ubicación precisa. La información \\
& debe ser considerada aproximadamente \\
& El elemento se representa gráficamente como un sistema \\
& genérico de objeto, tamaño, forma, ubicación y orientación \\
& aproximados. La información no grafica también es \\
& aproximada. Esta aproximación es respecto al volumen o \\
& espacio reservado. \\
& El elemento representa gráficamente como un objeto o sistema \\
& específico en términos de cantidad, tamaño, forma, ubicación y \\
& orientación. La información grafica se corresponde con la \\
& información gráfica. Las cantidades, dimensiones, formas, \\
& ubicación y orientación según lo diseñado se puede obtener \\
directamente o del elemento. & Igual al LOD 300, pero con representaciones se vinculan con \\
otros elementos del modelo cercano o adjunto y se incluyen los \\
partes tales como soporte o conexiones. \\
LOD 350 más la modelación. Estas representaciones se \\
modelan con la precisión y el detalle suficiente para su \\
fabricación e instalación.
\end{tabular}

Nota. Tomado de BIM Forum Chile (2017) 


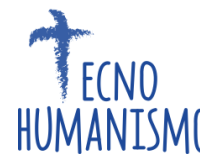

El desarrollo del modelo se desarrolló de la siguiente manera y siguiendo estos pasos:

- Levantamiento fotogramétrico y nube de puntos; Se utilizó un dron DJI Phantom 4 y para el diseño de la nube de puntos el software Autodesk Recap
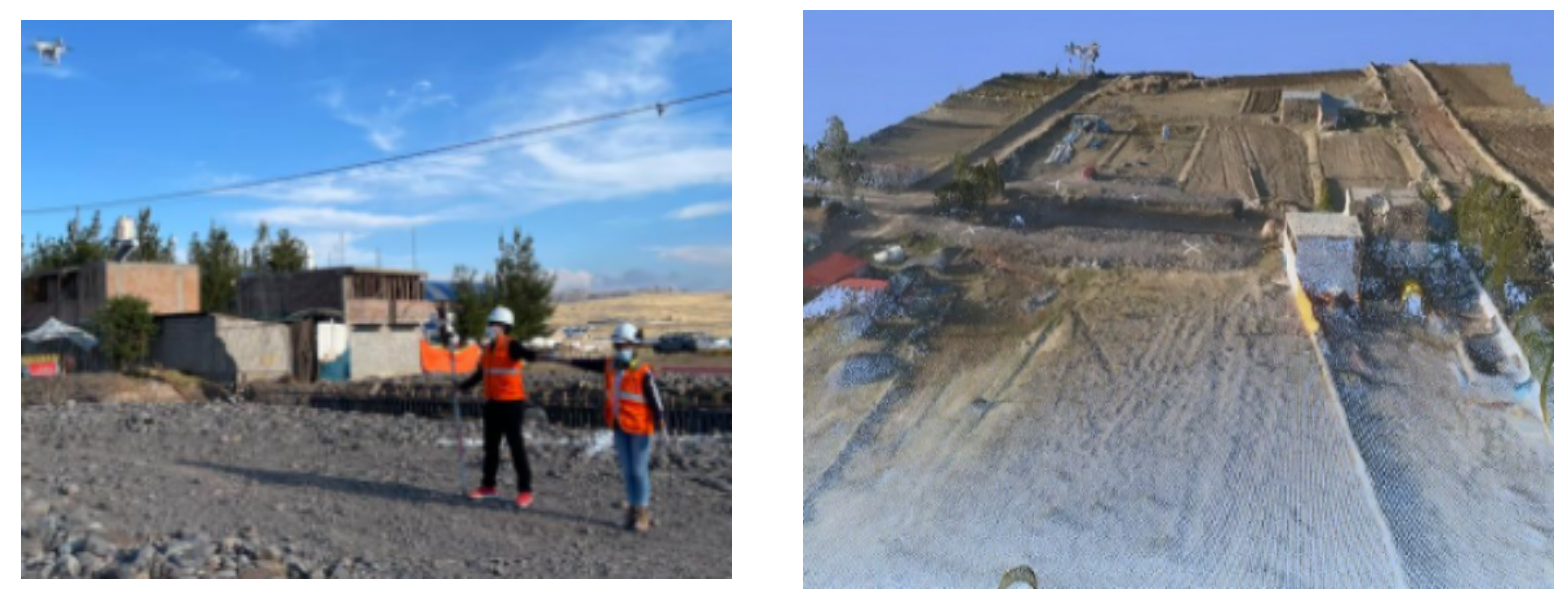

Figura 3 y 4. Levantamiento Dron y Nube de Puntos en Paucarcolla, Puno

- Alineamiento y diseño vial; se procesa la información de la nube de puntos y se realiza el diseño mediante el software Autodesk Civil3D.
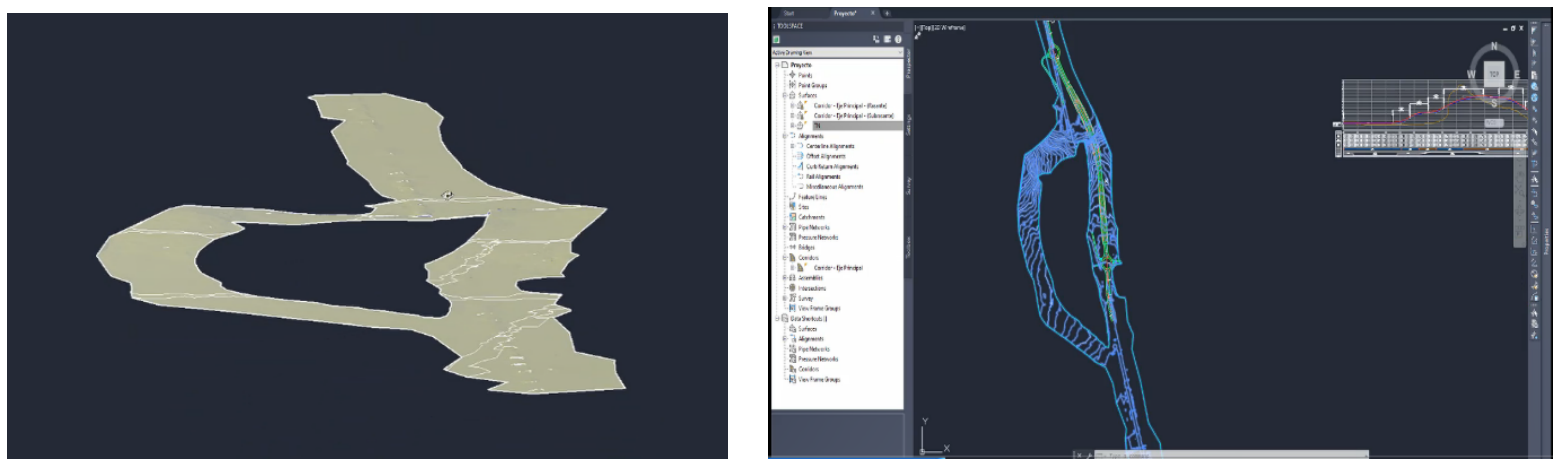

Figura 5 y 6. Diseño vial Intercambio Vial 
- Diseño y modelamiento 3D; con la información del diseño en Civil 3D y con la información de los planos del expediente técnico; se procede a diseñar y modelar en 3D utilizando el software Autodesk Revit.
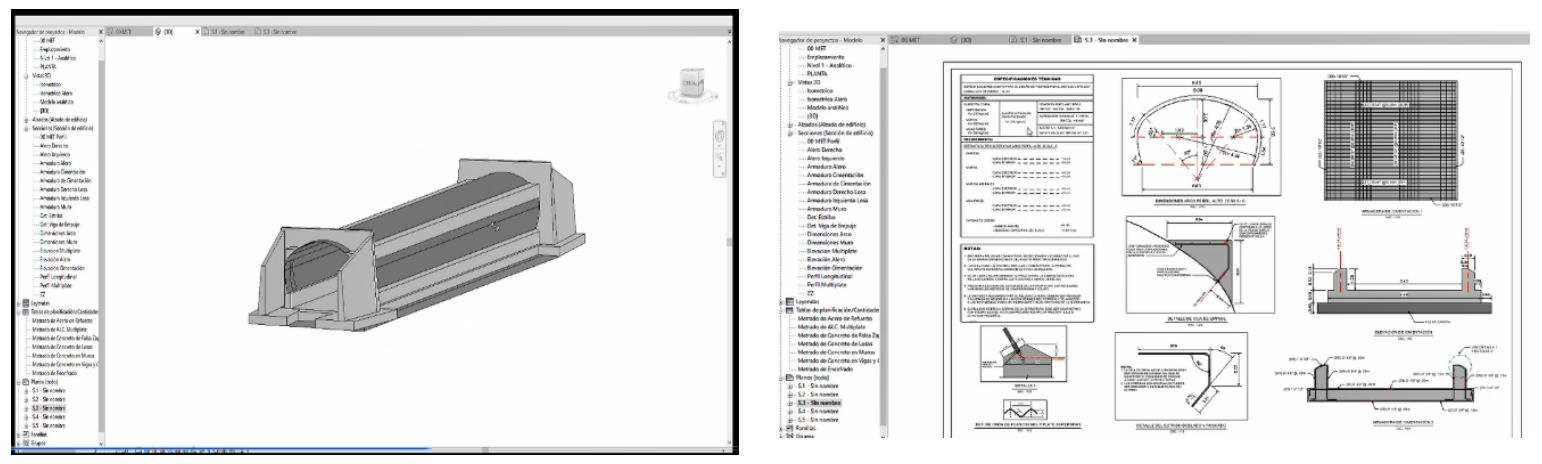

Figura 7 y 8. Diseño 3D y laminado en Revit

- Modelamiento y simulación; haciendo uso del software Autodesk Infraworks se hace una simulación del proceso constructivo con el fin de verificar la compatibilidad del diseño.

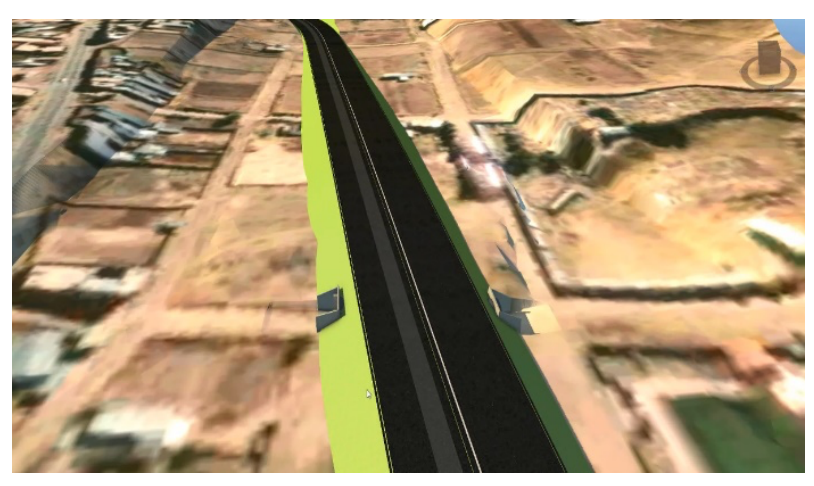

Figura 9. Modelamiento Infraworks 

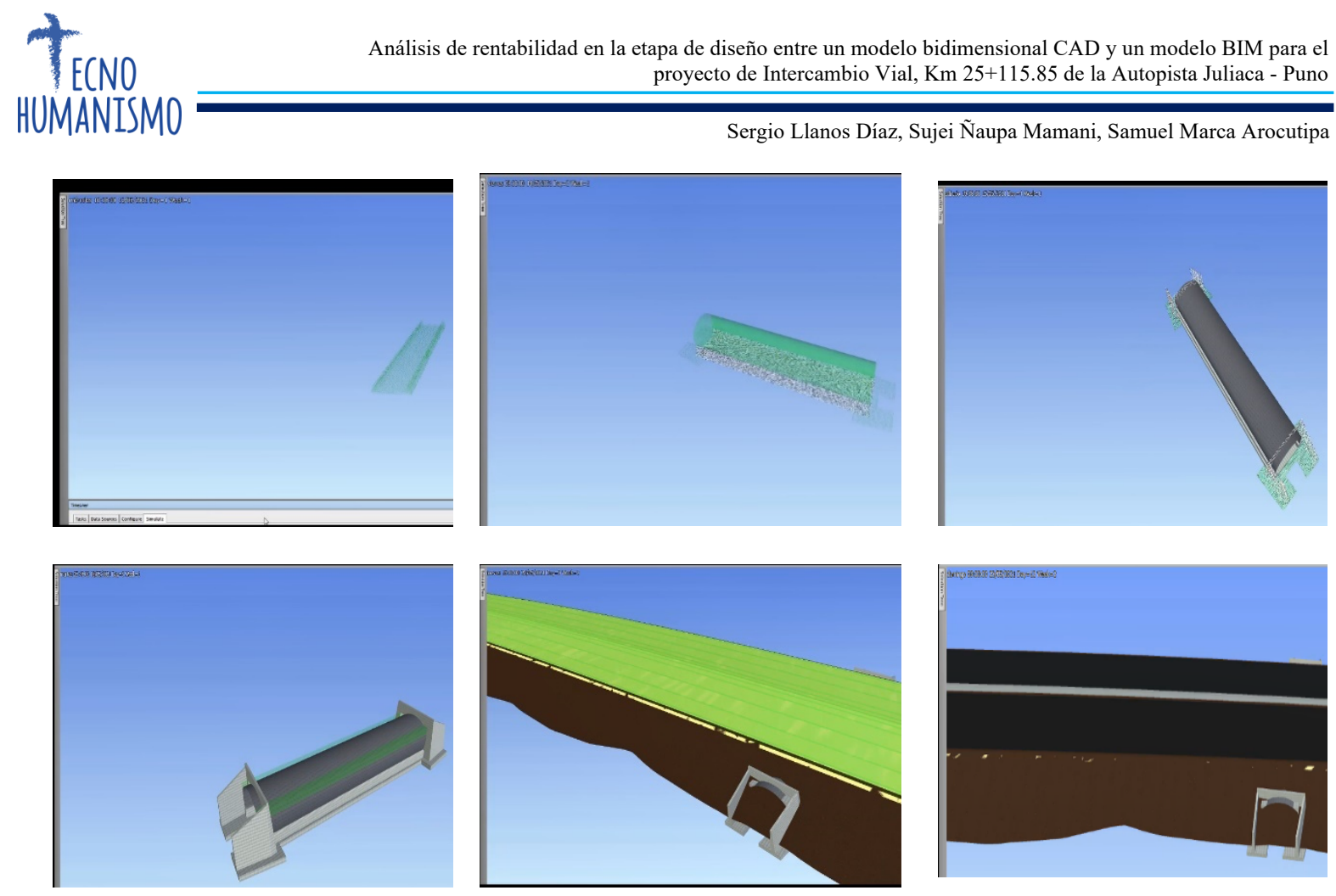

Figura 10. Simulación Navisworks

\subsubsection{Evaluar el rango de variabilidad del índice de rentabilidad entre el modelo convencional CAD y metodología BIM en las dimensiones de tiempos y costos del proyecto de intercambio vial $25+115.85$}

Se realizará un cuadro comparativo de valores obtenidos en base a la información de la metodología convencional CAD y BIM, donde se verificará el porcentaje de variabilidad en metrados no considerados en la metodología convencional, siendo en su gran mayoría en sobreanchos de la vía y parte de las curvas. 


\begin{tabular}{|c|c|c|c|}
\hline \multirow[b]{2}{*}{ CAPITULOS Y ACTIVIDADES } & \multirow[b]{2}{*}{ UND } & \multicolumn{2}{|c|}{ METODOLOGIA } \\
\hline & & $\begin{array}{c}\text { TRADICIONAL } \\
\text { 2D }\end{array}$ & $\begin{array}{l}\text { METODO } \\
\text { BIM }\end{array}$ \\
\hline \multicolumn{4}{|l|}{ CIMENTACIÓN } \\
\hline EXCAVACION A MAQUINA SIN RETIRO & M3 & 589,14 & 670,64 \\
\hline CONCRETO CICLOPEO 3000 PSI RELAC.60C/40P & M3 & 71,83 & 84,05 \\
\hline ZAPATA CONCRETO 3000 PSI INC. FORMALETA & M3 & 75,28 & 88,99 \\
\hline VIGA CIMIENTO ENLACE H=40 cms, INCLUYE SOLADO DE LIMPIEZA & M3 & 38,44 & 29,71 \\
\hline CONTRAPISO CONCRETO E=10CM $2.500 \mathrm{Psi}$ & M2 & 551,36 & 518,82 \\
\hline PISO RAMPA EN CONCRETO F'c= 3000 PSI & M2 & 12,00 & 10,00 \\
\hline ANDEN CONCRETO 10CM 3000 PSI (perimetral) & M2 & 547,00 & 574,55 \\
\hline RELLENO ROCA MUERTA COMPACTADO-SALTARIN & M3 & 158,74 & 130,64 \\
\hline SOLADO ESPESOR $E=0.05 \mathrm{M} \mathrm{F}^{\prime} \mathrm{c}=3000 \mathrm{PSI}-21 \mathrm{MPA}$ & M2 & 79,69 & 76,24 \\
\hline
\end{tabular}

Figura 11. Cuantificación inicial cantidades de obra metodología tradicional vs BIM

(Ramírez, 2018)

Tabla 4. Matriz de Rentabilidad de los métodos CAD y BIM.

\begin{tabular}{lcccc}
\hline \multirow{2}{*}{ Rentabilidad } & \multicolumn{2}{c}{ MÉTODO } & \multicolumn{2}{c}{ BIM } \\
\cline { 2 - 5 } & \multicolumn{2}{c}{ CAD } & PLAZO & COSTO (S/. \\
\cline { 2 - 5 } & PLAZO & COSTO $(\mathrm{S} /$ & PLH) \\
\hline Topografía & $(\mathrm{HH})$ & XH) & $(\mathrm{HH})$ & XH \\
\hline Mejoramiento de terreno & 24 & 40 & 20 & 30 \\
\hline Movimiento de tierras & 20 & 40 & 10 & 30 \\
\hline Cimentación & 30 & 40 & 25 & 30 \\
\hline Rellenos & 25 & 40 & 20 & 30 \\
\hline Plancha metálica corrugada & 45 & 40 & 40 & 30 \\
\hline Muros & 35 & 40 & 30 & 30 \\
\hline Sub base y bases & 26 & 40 & 16 & 30 \\
\hline Seguridad vial & 20 & 40 & 10 & 30 \\
\hline Pavimento & 40 & 40 & 35 & 30 \\
\hline
\end{tabular}


Utilizando la tabla 4 se calculó el costo total para cada método y se obtuvo la tabla $\mathrm{N}^{\circ} 5$

Tabla 5. Costo total de los métodos CAD y BIM.

\begin{tabular}{lcc}
\hline \multicolumn{3}{c}{ COSTO TOTAL } \\
\hline Topografía & $\mathrm{CAD}$ & $\mathrm{BIM}$ \\
Mejoramiento de terreno & $\mathrm{S} / .1200$ & $\mathrm{~S} / .600$ \\
Movimiento de tierras & $\mathrm{S} / .960$ & $\mathrm{~S} / .540$ \\
Cimentación & $\mathrm{S} / .800$ & $\mathrm{~S} / .300$ \\
Rellenos & $\mathrm{S} / .800$ & $\mathrm{~S} / .750$ \\
Plancha metálica corrugada & $\mathrm{S} / .100$ & $\mathrm{~S} / .600$ \\
Muros & $\mathrm{S} / .1800$ & $\mathrm{~S} / .1200$ \\
Sub base y bases & $\mathrm{S} / .1400$ & $\mathrm{~S} / .900$ \\
Seguridad vial & $\mathrm{S} / .1040$ & $\mathrm{~S} / .480$ \\
Pavimento & $\mathrm{S} / .800$ & $\mathrm{~S} / .300$ \\
\hline
\end{tabular}

Fuente. Elaboración propia

Para poder lograr un análisis de la información estadística, utilizaremos la Distribución T de Student. Según (García 2017) indica que la Distribución T de Student se utiliza para detectar la existencia de diferencias significativas entre las medias de una determinada variable cuantitativa en dos grupos de datos. También indica sobre:

Requisitos:

- Datos distribuidos según una distribución normal en cada grupo.

- Muestras independientes y menores a 30.

Procedimiento de cálculo

- El estadístico de prueba para una distribución t-student con $\mathrm{n} 1+\mathrm{n} 2-2$ grados de libertad es el siguiente:

$$
t_{p}=\frac{\bar{X}_{1}-\bar{X}_{2}}{S_{p *} \sqrt{\frac{1}{n_{1}}+\frac{1}{n_{2}}}} ; S_{p}=\sqrt{\frac{\left(n_{1}-1\right) S_{1}^{2}+\left(n_{2}-1\right) S_{2}^{2}}{n_{1}+n_{2}-2}}
$$

Donde: 


\section{$n_{1}$ y $n_{2}$ : Tamaños de las muestras 1 y 2 respectivamente \\ $\bar{X}_{1}$ y $\bar{X}_{2}:$ Medias de las muestras 1 y 2 respectivamente \\ $S_{1}^{2}$ y $S_{2}^{2}$ : Varianzas de las muestras 1 y 2 respectivamente}

A continuación, se mide la significación del estadístico $t_{p}$, comparando ese valor con el valor de un estadístico tabla que se obtiene mirando las tablas correspondientes.

Para identificar el $t_{\text {tabla }}$ que nos corresponde hemos de fijarnos en el número de colas que tiene nuestra hipótesis (una cola o dos colas), en el nivel de significación $(\alpha)$ con el que pretendemos rechazar la hipótesis nula; y en los grados de libertad del test $(\mathrm{n} 1+\mathrm{n} 2-2)$.

$$
\begin{aligned}
& \text { Si } t_{\mathrm{p}} \geq \mathrm{t}_{\text {tabla }}=>\text { se rechaza } H_{o} \text { y se acepta } H_{l} \\
& \text { Si } \mathrm{t}_{\mathrm{p}}<\mathrm{t}_{\text {tabla }}=>\text { se rechaza } H_{o} \text { y se rechaza } H_{l} .
\end{aligned}
$$

Tabla 6. Prueba T de Student

\section{Prueba T}

\begin{tabular}{|lrr|r|r|r}
\multicolumn{7}{c}{ Estadísticas de grupo } \\
\cline { 4 - 6 } & METODO & N & Media & $\begin{array}{c}\text { Desv. } \\
\text { Desviación }\end{array}$ & $\begin{array}{c}\text { Desv. Error } \\
\text { promedio }\end{array}$ \\
\hline COSTO & BIM & 9 & 680,00 & 319,257 & 106,419 \\
\cline { 2 - 6 } & CAD & 10 & 1400,00 & 333,200 & 105,367 \\
\hline
\end{tabular}

Fuente. Elaboración propia

\begin{tabular}{|c|c|c|c|c|c|c|c|c|}
\hline & & \multicolumn{2}{|c|}{$\begin{array}{c}\text { Prueba de Levene de igualdad } \\
\text { de varianzas }\end{array}$} & \multicolumn{5}{|c|}{ prueba t para la igualdad de medias } \\
\hline & & $\mathrm{F}$ & Sig. & $t$ & gl & Sig. (bilateral) & $\begin{array}{l}\text { Diferencia de } \\
\text { medias }\end{array}$ & $\begin{array}{c}\text { Diferencia de } \\
\text { error } \\
\text { estándar }\end{array}$ \\
\hline \multirow[t]{2}{*}{ COSTO } & $\begin{array}{l}\text { Se asumen varianzas } \\
\text { iguales }\end{array}$ &, 001 & .978 & $-3,331$ & 17 & ,004 & $-500,000$ & 150,114 \\
\hline & $\begin{array}{l}\text { No se asumen varianzas } \\
\text { iguales }\end{array}$ & & & $-3,339$ & 16,920 &, 004 & $-500,000$ & 149,757 \\
\hline
\end{tabular}

Tabla 7. Análisis de varianzas

Fuente. Elaboración propia 
Utilizando los datos provenientes de la tabla 4 se realizó el test de t-Student para verificar si las medias de los costos de ambos métodos son iguales o diferentes. Los resultados lo obtenemos con el software SPSS y obtuvimos que las medias de dichos métodos son estadísticamente diferentes y significativos con un $\mathrm{p}$-value $=0.004<0.05$, podemos ver también que la media del costo con el método BIM fue de S/ 680.00 y el costo con el método CAD fue de S/ 1180.00 (tabla 6 y 7).

\section{Discusion de resultados}

De acuerdo a los resultados encontrados se evidencia que la metodología BIM no solamente es mucho menos costosa por lo cual es mucho más rentable en relación a la metodología tradicional CAD lo cual se compara con los expuesto por Enshassi \& AbuHamra, 2016; Karlshøj, (2012) quien planteó que BIM permite una inimaginable gama de actividades a esto se agrega el hecho de que integra datos multidisciplinares para crear representaciones digitales detalladas que se administran en una plataforma abierta en la nube a fin de permitir la colaboración en tiempo real, esto igualmente es reforzado por Fernando (2017), quien menciona que el usar un software le proporciona una serie de ventajas al momento de hacer efectiva la planificación y ejecución de los proyectos de construcción.

Lamentablemente el uso de BIM en comparación con el CAD está mucho menos difundido en obras de infraestructura lo que no ha permitido su popularidad como en otras áreas de la ingeniería civil, donde el BIM aporta como anteriormente se mencionó no solo aspectos referidos a un menor costo, sino que en el tema de los tiempos, el uso de BIM aminora los plazos en cada una de las fases tal y como se encontró en los resultados, donde cimentación hasta mejoramiento del terreno se hace en un corto plazo hasta y las variaciones fluctúan de forma bastante interesante, en el caso de cimentación al utilizar BIM se desarrolla en un $16.6 \%$ menor con respecto a CAD y en mejoramiento de terreno se vuelve mucho más significativo cuando al usar BIM el plazo disminuye hasta en un 38\% en comparación al usar CAD. Esto es comparable con los estudios realizados por Garzón et al., (2018) quien estableció que la metodología BIM aporta a la gestión de proyectos en función de tiempos y los costos, esto es igualmente confirmado por la opinión de Medina y Salomón (2019) quien menciona que BIM, es de hecho una metodología que contribuye en la reducción del tiempo de trabajo para estimar los metrados para costos, porque reduce los días de trabajo y 
lógicamente esto genera un impacto económica en las obras, por tanto tiene incidencia directa en la rentabilidad de los proyectos realizados.

\section{Conclusión}

De esta manera concluir que entre las 02 metodologías en cuanto a la rentabilidad del proyecto el método BIM tiene un costo más bajo que el CAD por lo que es más recomendable utilizar este tipo de método en la estructura formada por planchas de acero corrugado galvanizado (Multiplate MP152), cimentación y muros de concreto armado, relleno estructural y pavimento flexible del proyecto de Intercambio Vial, Km 25+115.85 de la Autopista Juliaca - Puno.

\section{Referencias}

ASIDEK. (12 de diciembre de 2016). La situación del BIM en el mundo evoluciona. ASIDEK. https://www.asidek.es/la-situacion-del-bim-mundo

BIM Forum Chile (2017). Guía inicial para implementar BIM en las organizaciones. Corporación de Desarrollo Tecnológico - Cámara Chilena de la Construcción. https://www.bimforum.cl/wp-content/uploads/2017/07/Gu\%c3\%ada-inicial-paraimplementar-BIM-en-las-organizaciones-versi\%c3\%b3n-imprenta.pdf

Borja, S. A. (18 de noviembre de 2015). Qué es Revit o mejor, qué es BIM. Espacio BIM. https://www.espaciobim.com/que-es-revit/

Chavarría, E. (2018). La metodología BIM para optimizar el diseño de la carretera Luricocha-Pacchancca, Ayacucho 2018. Universidad César Vallejo. https://repositorio.ucv.edu.pe/handle/20.500.12692/22807

Contreras, J., Garzón, Y., Gómez, A. \& Misie, R. (2018). Integración entre building information modeling $\mathrm{y}$ project management institute como propuesta metodológica para la gestión de proyectos. Ingeniería, vol. 22, núm. 3, pp. 1-16. https://www.redalyc.org/journal/467/46759491001/html/

Enshassi, A., \& AbuHamra, L. (2016). Investigación de las funciones del modelado de la información de construcción en la industria de la construcción en Palestina. Revista Ingenieria de Construccion, 31(2), 127-138. https://doi.org/10.4067/S0718$\underline{50732016000200006}$ 
EquipoPVP. (17 de agosto de 2018). ProgramasvirtualesPC. Obtenido de https://www.programasvirtualespc.net/software/autocad-civil-3d-2019-0-1espanole-ingles-construccion-y-diseno-en-ingenieria-civil/es.BIM

Fernando. (12 de 04 de 2017). Autodesk Latam. Obtenido de http://blogs.autodesk.com/latam/2017/04/12/autodesk-infraworks-colaboracionyvisualizacion-a-la-vanguardia/

García, B. (2017). Efecto de la Fibra de Vidrio en las propiedades mecánicas del Concreto $F^{\prime} C=210 \mathrm{~kg} / \mathrm{cm} 2$ en la ciudad de puno. Universidad Nacional del Altiplano.

https://repositorio.unc.edu.pe/bitstream/handle/UNC/656/T\%20690\%20P739\%20 2014.pdf? sequence $=1 \&$ isAllowed $=y$

Huancas, E. \& Torres, H. (2020). METODOLOGÍA BIM PARA CICLOS DE DESARROLLO DE PROYECTOS INMOBILIARIOS, LAMBAYEQUE. 2018. Universidad Señor de Sipán. Pimentel, Perú. https://repositorio.uss.edu.pe/bitstream/handle/20.500.12802/6997/Huancas\%20Ti neo $\% 20$ Edwin $\% 20 \% 26 \% 20$ Torres $\% 20$ Pedraza $\% 20$ Heggel.pdf?sequence $=1 \&$ isA1 lowed $=\mathrm{y}$

Ministerio de Transportes y Comunicaciones (2016). Informe de Modificación de Ingeniería de la Variante de Paucarcolla correspondiente a la Construcción de la Autopista Puno - Juliaca. MTC; Covisur.

Karlshøj, J. (2012). Not just CAD ++. BIM Journal, 3(28), 39-42.

Medina, P. \& Salomón, N. (2019). Evaluación de la estimación de metrados para los costos de la partida de arquitectura de una obra retail en Lima en el 2019 con la implementación BIM. Universidad Tecnológica del Perú. Lima, Perú. https://repositorio.utp.edu.pe/bitstream/handle/20.500.12867/3435/Pablo\%20Medi na_Nataly\%20Salomon_Trabajo\%20de\%20Investigacion_Bachiller_2019.pdf?se quence $=1 \&$ isAllowed $=\mathrm{y}$

Noor, B. A., \& Yi, S. (2017). Building Information Modeling in Construction Phase. International Journal of Engineering and Innovative Technology (IJEIT), 33(10), 36-44. https://doi.org/10.17605/OSF.IO/TB6U9

Monfort, C. (2015) Impacto del BIIM en la gestión del proyecto y la obra de arquitectura. Universidad politécnica de Valencia. Escola Técnica de Arquitectura. 
https://docplayer.es/8512882-Impacto-del-bim-en-la-gestion-del-proyecto-y-laobra-de-arquitectura-un-proyecto-con-revit.html

Ramírez, J. (2018). Comparación entre metodologías building information modeling (BIM) y metodologías tradicionales en el cálculo de cantidades de obra y elaboración de presupuestos. Caso de estudio: edificación educativa en Colombia. Universidad Distrital Francisco José de Caldas. Bogotá, Colombia.

Ureña, V. (20 de junio de 2020). Infrastructure Technical Specialist en Autodesk [Discurso principal]. Conferencia de las Naciones Unidas, Autodesk Spain \& Portugal. 